Natural Hazards and Earth System Sciences (2003) 3: 353-365

(C) European Geosciences Union 2003

Natural Hazards and Earth System Sciences

\title{
Tsunami excitation by inland/coastal earthquakes: the Green function approach
}

\author{
T. B. Yanovskaya ${ }^{1}$, F. Romanelli ${ }^{2}$, and G. F. Panza ${ }^{2,3}$ \\ ${ }^{1}$ Institute of Physics, St. Petersburg University, Petrodvoretz, 198904 St. Petersburg, Russia \\ ${ }^{2}$ Dipartimento di Scienze della Terra, Via E. Weiss 4, 34127 Trieste, Italy \\ ${ }^{3}$ The Abdus Salam International Centre for Theoretical Physics, SAND Group, Strada Costiera 11, Trieste, Italy
}

Received: 8 October 2002 - Accepted: 19 November 2002

\begin{abstract}
In the framework of the linear theory, the representation theorem is derived for an incompressible liquid layer with a boundary of arbitrary shape and in a homogeneous gravity field. In addition, the asymptotic representation for the Green function, in a layer of constant thickness is obtained. The validity of the approach for the calculation of the tsunami wavefield based on the Green function technique is verified comparing the results with those obtained from the modal theory, for a liquid layer of infinite horizontal dimensions. The Green function approach is preferable for the estimation of the excitation spectra, since in the case of an infinite liquid layer it leads to simple analytical expressions. From this analysis it is easy to describe the peculiarities of tsunami excitation by different sources. The method is extended to the excitation of tsunami in a semiinfinite layer with a sloping boundary. Numerical modelling of the tsunami wavefield, excited by point sources at different distances from the coastline, shows that when the source is located at a distance from the coastline equal or larger than the source depth, the shore presence does not affect the excitation of the tsunami. When the source is moved towards the coastline, the low frequency content in the excitation spectrum decreases, while the high frequencies content increases dramatically. The maximum of the excitation spectra from inland sources, located at a distance from the shore like the source depth, becomes less than $10 \%$ of that radiated if the same source is located in the open ocean.
\end{abstract}

The effect of the finiteness of the source is also studied and the excitation spectrum is obtained by integration over the fault area. Numerical modelling of the excitation spectra for different source models shows that, for a given seismic moment, the spectral level, as well as the maximum value of the spectra, decreases with increasing fault size.

When the sources are located in the vicinity of a shore, the synthetic mareograms calculated at distances greater than the source depth show that the maximum tsunami amplitude

Correspondence to: F. Romanelli

(romanel@dst.univ.trieste.it) decays with decreasing source-to-shore distance. The rate of decay is dependent on the dip, length and depth of the fault. The tsunami intensity, defined as maximum peak-topeak amplitude, decays with the inland distance of the source from the coast. At an inland distance equal to the source depth, it becomes 4-5 times less than that from a source in the open ocean. If the source is located under the coastline, the intensity of tsunami is approximately the same as for oceanic sources.

\section{Introduction}

Two approaches exist for modelling tsunami generation by earthquakes: one is based on the modal theory (Pod'yapolsky, 1968; Ward, 1980; Comer, 1984a; Panza et al., 2000), another (referred in some papers as 'traditional') proceeds from solving the hydrodynamic equations with the boundary conditions at the ocean floor which correspond to static displacements caused by the earthquake source (e.g. Hammack, 1973; Lee and Chang, 1980; Okal, 1982; Comer, 1984b). As indicated by Comer (1984b), the former approach assumes the ocean and solid Earth to be fully coupled, whereas according to the latter they are partially coupled. The term "partially coupled" means that the solid earth affects the movement of the liquid, but the liquid does not affect the displacement in the solid Earth: it is assumed to be the same as in absence of the liquid layer. Comer (1984b) has shown that the two approaches are nearly identical.

Though the modal theory gives a solution corresponding to the exact boundary conditions, and it may be easily extended to models with slightly varying thickness of the water layer, it can be applied only when a source is located under the ocean, far away from a coast. However, there are indications that sources near a coastline and even under the land, may cause intense tsunami waves. The most typical example is the Alaskan earthquake of $1964\left(M_{w}=9.2\right)$ that generated a tsunami concentrated along the Californian and Chilean Coasts, where the run-up heights were of the order of 


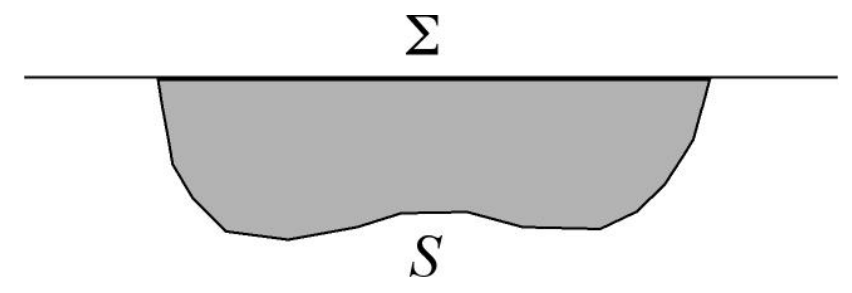

Fig. 1. Model of a liquid basin over a solid half-space. $S$ is the liquid-solid boundary, $\Sigma$ is the free surface of the water layer.

$4 \mathrm{~m}$ due to a beaming effect (e.g. Ben-Menhaem and Rosenman, 1972; Johnson et al., 1996). Besides distant tsunamis that have travelled far from the origin of the earthquake, the analysis of how earthquake source parameters affect the excitation of the tsunami wavefield has a great importance in the study of local tsunamis (e.g. Titov and Synolakis, 1997; Ortiz et al., 1998; Geist, 1999).

For the analysis of such a case the ideas of the traditional approach may be effective, though no one of the existing versions of the method can be applied directly. A suitable approach may be that based on the Green function technique, as proposed firstly by Kajiura (1963) for the analysis of tsunamis excited by an impulsive source (displacement, pressure, deformation) distributed within a finite area of the bottom in an infinite water layer of constant thickness. It is well known that the problems, in which the waves are generated due to given displacements at a closed surface, are effectively solved by the use of this approach. The exact solution for the Green function in the liquid layer is represented in an integral form (Kajiura, 1963). Therefore, to simplify the problem, we have to use the asymptotic representation valid in the far-field and, consequently, we can estimate only the far-field tsunami (source receiver distances of the order of tens of $\mathrm{km}$, or more). The far field estimate allows us to draw useful conclusions about (a) the influence of the fault parameters on the excitation of the tsunami wavefield, that turn out to be in good agreement with previous studies (e.g. Yamashita and Sato, 1974; Kajiura, 1981; Ward, 1982); (b) the intensity of a tsunami generated by a seismic source near to the coast, which is useful for the assessment, in a quick and efficient way, of the tsunami hazard.

\section{Representation theorem for tsunami waves}

We consider a basin filled by an incompressible liquid in contact with a solid along an interface $S$ of arbitrary shape and with horizontal free surface $\Sigma$ (see Fig. 1). The equation of motion for the liquid, using the linear theory, can be written as:

$\nabla p(\mathbf{x}, t)+\rho_{f} \frac{\partial^{2} \mathbf{u}(\mathbf{x}, t)}{\partial t^{2}}=\mathbf{F}(\mathbf{x}, t)$,

where $p(\mathbf{x}, t)$ is the pressure, $\mathbf{u}(\mathbf{x}, t)$ is the displacement, and $\rho_{f}$ the is density of the fluid. For harmonic waves of fre- quency $\omega$ this equation may be rewritten, omitting the time dependence, in the form:

$\nabla p(\mathbf{x})-\rho_{f} \omega^{2} \mathbf{u}(\mathbf{x})=\mathbf{F}(\mathbf{x})$.

If a unit point force directed along the $q$-axis is placed at a point $\mathbf{x}_{0}$, we obtain the equation for the Green function $\mathbf{u}^{q}\left(\mathbf{x}, \mathbf{x}_{0}\right)$ :

$\nabla p\left(\mathbf{u}^{q}\right)-\rho_{f} \omega^{2} \mathbf{u}^{q}=\mathbf{e}_{q} \delta\left(\mathbf{x}-\mathbf{x}_{0}\right)$.

For simplicity we shall denote the pressure corresponding to the Green function as $p^{q}$, i.e. $p^{q}=p\left(\mathbf{u}^{q}\right)$.

The wave field $\mathbf{U}(\mathbf{x})$, and the associated pressure $P=$ $p(\mathbf{U})$, caused only by a displacement (or pressure) on the surface $S+\Sigma$, in the absence of body forces, satisfies the equation:

$\nabla P-\rho_{f} \omega^{2} \mathbf{U}=0$.

Multiplying (3) by $\mathbf{U}$, (4) by $\mathbf{u}^{q}$, subtracting (3) from (4), and integrating over the volume $\Omega$ bounded by $S+\Sigma$, we represent the $q$-component of the displacement as:

$U_{q}\left(\mathbf{x}_{0}\right)=\int_{\Omega}\left[\left(\mathbf{U}, \nabla p^{q}\right)-\left(\mathbf{u}^{q}, \nabla P\right)\right] d \Omega$,

where (,) stands for the scalar product.

Since the liquid is incompressible, $\operatorname{div} \mathbf{U}=\operatorname{div} \mathbf{u}^{q}=0$, and the integral (5) may be rewritten as:

$$
\begin{aligned}
& U_{q}\left(\mathbf{x}_{0}\right)=\int_{\Omega}\left[\operatorname{div}\left(p^{q} \mathbf{U}\right)-\operatorname{div}\left(P \mathbf{u}^{q}\right)\right] d \Omega= \\
& \iint_{S+\Sigma}\left(p^{q} U_{n}-P u_{n}^{q}\right) d S .
\end{aligned}
$$

At the free surface $\Sigma$ the boundary condition is $p=$ $-\rho_{f} g u_{n}$, so that the integral over $\Sigma$ vanishes, and finally we obtain:

$U_{q}\left(\mathbf{x}_{0}\right)=\iint_{S}\left(p^{q} U_{n}-P u_{n}^{q}\right) d S$,

that is a general form of the representation theorem for an incompressible liquid.

If the Green function is chosen so that $u_{n}^{q}(S)=0$, then (7) simplifies to:

$U_{q}\left(\mathbf{x}_{0}\right)=\iint_{S} p^{q} U_{n} d S$.

\section{Green function for a liquid layer of constant thickness}

The most important characteristic of the tsunami wave field is the vertical displacement at the liquid surface. Therefore we focus on the Green function corresponding to a vertical point force $(q=z)$ located at a point $\mathbf{x}_{0}=\left(0,0, z_{0}\right)$, the 


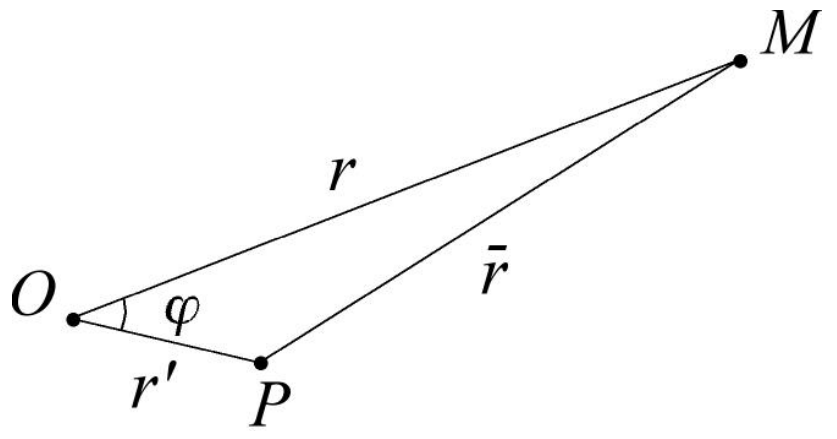

Fig. 2. Reference system: $O$ is the projection of a point source on the $x y$-plane (see Fig. 3), which is taken as the reference system, $M$ is the observation point, $P$ is the current point on the $x y$-plane.

$z$-axis being directed downward (to obtain the displacement at free surface we take $z_{0}=0$ ). The thickness of the liquid layer is $H$, and the vertical and horizontal displacements are denoted by $W$ and $U$, respectively. Though the expression for $p^{z}\left(\mathbf{x} ; \mathbf{x}_{0}\right)$ can be obtained, after some manipulations, as the Fourier transform of the corresponding equation given by Kajiura (1963) for the time-dependent Green function, we prefer to derive it here in the following alternative way. We solve the equation of motion for the Green function in cylindrical coordinates, and we represent the 3-D delta-function in the right-hand side of (3) as:

$\frac{\delta\left(z-z_{0}\right)}{2 \pi} \int_{0}^{\infty} J_{0}(k r) k d k$.

A solution for the pressure can be written in the form of the Fourier-Bessel integral:

$P(r, z)=\int_{0}^{\infty} P(z) J_{0}(k r) d k$,

where $P(z)$ satisfies the equation:

$\frac{d^{2} P}{d z^{2}}-k^{2} P=0$

everywhere except at $z=z_{0}$, while at $z=z_{0} P(z)$ has a jump equal to $k / 2 \pi$. The solution $P(z)$ should satisfy the boundary conditions:

$P(0)=-\rho_{f} g W(0), \quad W(H)=0$

and

$P\left(z_{0}^{+}\right)-P\left(z_{0}^{-}\right)=k / 2 \pi, \quad W\left(z_{0}^{+}\right)=W\left(z_{0}^{-}\right)$.

It is easy to show that the solution of (10b) with the boundary conditions $(11 \mathrm{a}-11 \mathrm{~b})$ is:

$P(z, k)=\frac{k\left(\cosh k z_{0}-\frac{g k}{\omega^{2}} \sinh k z_{0}\right)}{2 \pi\left(-\cosh k H+\frac{g k}{\omega^{2}} \sinh k H\right)} \cosh k(H-z)$

and

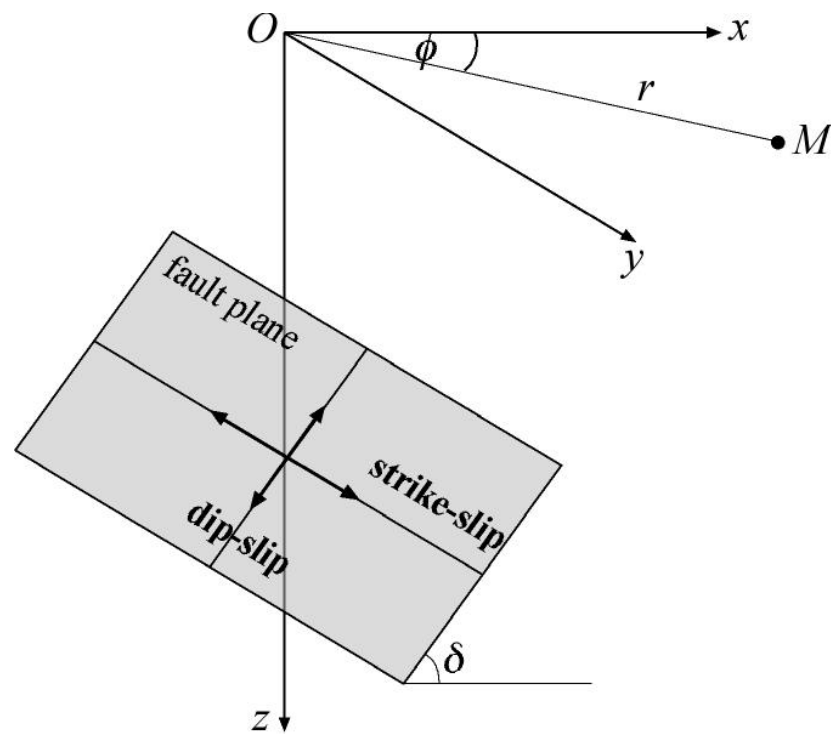

Fig. 3. Geometry of the fault plane. Arrows show slip direction for strike-slip and dip-slip mechanisms. $M$ is the observation point.

$P(z, r)=\int_{0}^{\infty} \frac{k\left(\cosh k z_{0}-\frac{g k}{\omega^{2}} \sinh k z_{0}\right)}{2 \pi\left(-\cosh k H+\frac{g k}{\omega^{2}} \sinh k H\right)} \cosh k(H-z) J_{0}(k r) d k$.

The integral (12) is estimated as a residue in the root of the denominator:

$\tanh k H=\frac{\omega^{2}}{g k}$,

that is the well known dispersion equation for an incompressible liquid over a rigid half-space. For $z_{0}=0$

$P(r, z)=-\frac{i H_{0}^{(2)}(k r)}{2} \frac{k \sinh k H}{H+\frac{g}{\omega^{2}} \sinh ^{2} k H} \cosh k(H-z)$,

that is the Green function $p^{z}(r, z ; 0,0)$.

Using the asymptotic expression of the Hankel function $\left(H_{0}^{(2)}(k r)\right)$, valid for $k r \gg 1$, we obtain:

$p^{z}(r, z ; 0 ; 0) \cong \exp (-i \pi / 4) \exp (-i k r)$

$\sqrt{\frac{1}{8 \pi k r}} \frac{2 k \sinh k H}{H+\frac{g}{\omega^{2}} \sinh ^{2} k H} \cosh k(H-z)$.

If $k r \geq 5$, the far-field expression (15) guarantees accuracy of at least 3 significant digits (e.g. Panza. et al., 1973). Considering the characteristic frequencies of the tsunami wavefield, this condition corresponds to a limit distance of the order of tens of $\mathrm{km}$ if one wants to maintain graphical accuracy in the mareogram calculation for hazard studies.

\section{Tsunami excitation in an infinite water layer}

The representation theorem (8) contains the normal displacement at the liquid-solid interface. We check the validity of 
(8) considering an infinite water layer of constant thickness, since, for this model, the results may be compared with those obtained on the basis of modal theory (Panza et al., 2000).

As in all the other studies, we replace the displacement the of bottom, which should satisfy the Eq. (4) and the continuity of normal stress and displacement at the bottom, by the static displacement $(\omega=0)$ at the surface, supposed free, of the solid half-space. This problem has been solved by different authors (Maruyama, 1964; Okada, 1985; Yamasaki, 1987), though usually the equations given by Okada (1985) are used. The equations needed in this paragraph, for the vertical and horizontal displacements from vertical and horizontal point forces, are given in Appendix A. It is easier to obtain solutions for tsunami wave fields for these two simple sources, and to determine the fields for double-couples by differentiation of the fields, rather than to obtain the bottom displacement directly for a double-couple.

Introducing (15) and (A1) in (8), we obtain the following equation for the vertical displacement of the water surface due to a vertical force:

$$
\begin{aligned}
& W(r, 0)=\frac{\exp (-i \pi / 4)}{4 \pi \rho \beta^{2}} \frac{2 k \sinh k H}{H+\frac{g}{\omega^{2}} \sinh ^{2} k H} \int_{0}^{2 \pi} \int_{0}^{\infty} \sqrt{\frac{1}{8 \pi k \bar{r}}} \\
& \left(\frac{K_{1}}{\sqrt{h^{2}+r^{\prime 2}}}+\frac{h^{2}}{\left(h^{2}+r^{\prime 2}\right)^{3 / 2}}\right) \exp (-i k \bar{r}) r^{\prime} d r^{\prime} d \varphi,
\end{aligned}
$$

where $K_{1}$ is defined in Appendix A, and the other notations are defined in Fig. 2.

We shall obtain the tsunami field at large distance $r$ from the source (far-field): the displacement of the bottom, which is involved in the integrand of (16), differs noticeably from zero in the vicinity of the source, i.e. for of $r^{\prime} \ll r$. If this condition is satisfied, it corresponds to $r \gg h$, and it has to be taken into account together with $k r \geq 5$ to assess the minimum distance at which the tsunami wavefield can be calculated with the desired accuracy. The extended or finite source is treated as a discrete sum of point sources.

Therefore one can use the approximation:

$\bar{r} \cong r-r^{\prime} \cos \varphi$

and it is sufficient to replace $\bar{r}$ by (17) only in the phase factor in the integrand, while in the amplitude factor we may assume $\bar{r}=r$. Thus (16) may be written as:

$$
\begin{aligned}
& W(r, 0)=\frac{\exp (-i \pi / 4) \exp (-i k r)}{4 \pi \rho \beta^{2}} \frac{2 k \sinh k H}{H+\frac{g}{\omega^{2}} \sinh ^{2} k H} \sqrt{\frac{1}{8 \pi k r}} \\
& \int_{0}^{2 \pi} \int_{0}^{\infty}\left(\frac{K_{1}}{\sqrt{h^{2}+r^{\prime 2}}}+\frac{h^{2}}{\left(h^{2}+r^{\prime 2}\right)^{3 / 2}}\right) \exp \left(i k r^{\prime} \cos \varphi\right) r^{\prime} d r^{\prime} d \varphi
\end{aligned}
$$

and taking into account that

$$
\int_{0}^{2 \pi} \exp \left(i k r^{\prime} \cos \varphi\right) d \varphi=2 \pi J_{0}\left(k r^{\prime}\right)
$$

we obtain

$$
\begin{aligned}
& W(r, 0)=\frac{\exp (-i \pi / 4) \exp (-i k r)}{\rho \beta^{2}} \frac{k \sinh k H}{H+\frac{g}{\omega^{2}} \sinh ^{2} k H} \sqrt{\frac{1}{8 \pi k r}} \\
& \int_{0}^{\infty} J_{0}\left(k r^{\prime}\right)\left(\frac{K_{1}}{\sqrt{h^{2}+r^{\prime 2}}}+\frac{h^{2}}{\left(h^{2}+r^{\prime 2}\right)^{3 / 2}}\right) r^{\prime} d r^{\prime}
\end{aligned}
$$

or finally

$$
\begin{aligned}
& W(r, 0)=\frac{\exp (-i \pi / 4) \exp (-i k r)}{\rho \beta^{2}} \sqrt{\frac{1}{8 \pi k r}} \\
& \frac{\sinh k H}{H+\frac{g}{\omega^{2}} \sinh ^{2} k H}\left(k h+K_{1}\right) \exp (-k h) .
\end{aligned}
$$

In a similar way we may obtain the vertical displacement of the water surface due to a horizontal point force directed along the x-axis. Equations (15) and (A3) are introduced in (8), and recalling that:

$$
\int_{0}^{2 \pi} \cos \varphi \exp \left(i k r^{\prime} \cos \varphi\right) d \varphi=2 \pi i J_{1}\left(k r^{\prime}\right)
$$

we obtain the following expression for the tsunami displacement, due to a horizontal force, at a receiver placed at azimuth $\phi$, measured from the $\mathrm{x}$-axis $\left(\cos \phi=\frac{x}{r}\right)$ :

$W(r, 0)=i \frac{\exp (-i \pi / 4) \exp (-i k r)}{\rho \beta^{2}} \sqrt{\frac{1}{8 \pi k r}}$
$\frac{\sinh k H}{H+\frac{g}{\omega^{2}} \sinh ^{2} k H}\left(k h-K_{2}\right) \exp (-k h) \cos \phi$.

Equations (19) and (20) may be compared with those resulting from the modal theory. It is convenient to write the asymptotic equation for the frequency spectrum of any surface-wave mode (including the tsunami mode) in the following form (Levshin et al., 1989; Panza et al., 2000):

$$
\begin{aligned}
& \mathbf{U}(r, \phi, z, \omega, t)=\frac{\exp (-i \pi / 4)}{\sqrt{8 \pi}} \frac{\exp [i(\omega t-k r)]}{\sqrt{k r}} \\
& \frac{\Psi(h, \phi, \omega)}{\sqrt{c u I_{0}}} \frac{\mathbf{u}(z, \omega)}{\sqrt{c u I_{0}}},
\end{aligned}
$$

where $c$ and $u$ are phase and group velocities respectively, $\mathbf{u}(z, \omega)$ is the eigenvector of the given mode, $I_{0}$ is the energy integral $\int_{0}^{\infty} \rho|\mathbf{u}(z, \omega)|^{2} d z$, and $\phi$ is the strike-receiver angle. The second factor in (21) describes the effect of propagation, the third is the so-called excitation factor $\chi=\frac{\Psi(h, \phi, \omega)}{\sqrt{\mathrm{cu} I_{0}}}$, and the fourth one is the receiver factor. The excitation factor depends on the source mechanism (i.e. on the radiation pattern, which has been identified by $\chi$ in Appendix C of Panza et al., 2000) and on the spectrum of the source time function. Usually, in this expression the spectrum of the source time function is omitted (or, equivalently, is assumed as the deltafunction spectrum), so that the excitation function expresses the capability of a source with a certain mechanism to excite 


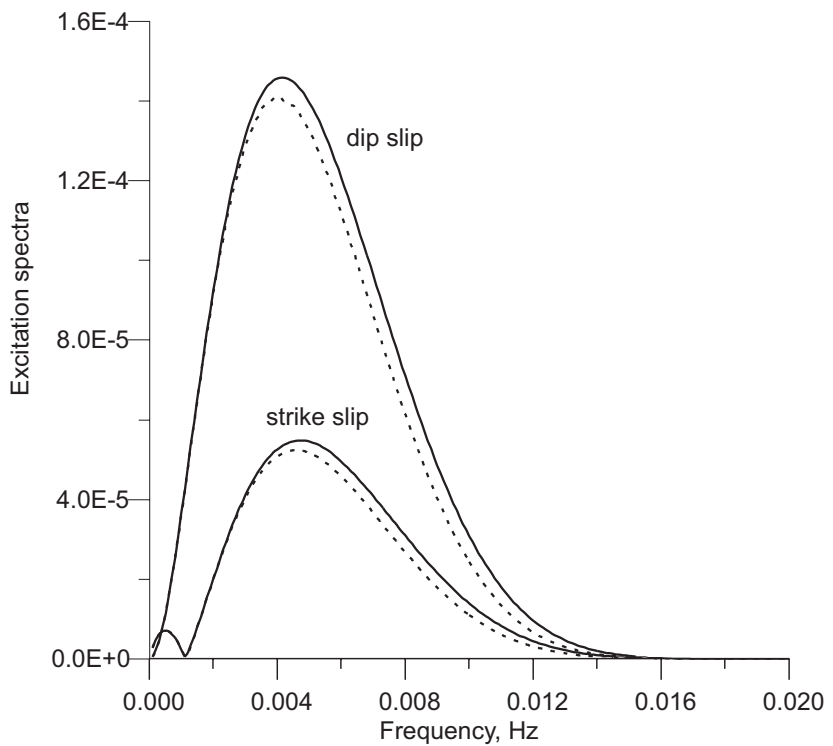

Fig. 4. Comparison of the excitation spectra calculated from: (a) the modal theory (solid lines) and (b) the Green function approach (dashed lines). The excitation spectra are calculated for a thickness of the water layer equal to $2 \mathrm{~km}$, and a source depth equal to $10 \mathrm{~km}$. The dip-slip and strike-slip mechanisms are both on a normal fault $\left(\delta=90^{\circ}\right)$. The spectra are calculated for the maximum of radiation, i.e. $\phi=90^{\circ}$ for the dip slip source, and $\phi=45^{\circ}$ for the strike slip one.

the mode under consideration. To extract the excitation factor from (19) and (20) it is sufficient to eliminate the constant and the propagation factors, and to divide the remaining by the receiver factor $\frac{W(0, \omega)}{\sqrt{\text { cuI }} \text {. }}$.

For a model formed by an incompressible water layer over an elastic half-space, the eigenfunctions and the energy integral are given in Appendix B, and we can write:

$\frac{W(0, \omega)}{\sqrt{c u I_{0}}}=\frac{\sqrt{k \sinh k H}}{\sqrt{\rho_{f} c u}}$.

Then the excitation factor obtained with the Green function technique for a vertical force is given by:

$\chi_{v}=F(k)\left(k h+K_{1}\right) \exp (-k h)$

and for a horizontal force directed along the $\mathrm{x}$-axis

$\chi_{h}=i F(k)\left(k h-K_{2}\right) \exp (-k h) \cos \phi$,

where $F(k)$ is given by:

$F(k)=\frac{\sqrt{\sinh k H} \sqrt{\rho_{f} c u}}{\rho \beta^{2}\left(H+\frac{g}{\omega^{2}} \sinh ^{2} k H\right) \sqrt{k}}$.

The excitation functions for any double-couple may be obtained by differentiating the expressions (23) and (24) with respect to the corresponding coordinates.

Let the geometry of a fault be defined by the strike direction along the y-axis and by the dip angle $\delta ; \phi$ is the angle between the x-axis and direction to the receiver (Fig. 3).
Then the excitation function is:

$$
\begin{aligned}
& \chi_{d s}(h, \phi)=F k\{2 i k h \cos 2 \delta \cos \phi+\sin 2 \delta \\
& \left.\left(2 k h+\sin ^{2} \phi\left(K_{2}-k h\right)\right)\right\} \exp (-k h)
\end{aligned}
$$

for a dip-slip source and:

$\chi_{s s}(h, \phi)=F k\{-2 i k h \sin \phi \cos \delta$

$\left.+\sin 2 \phi \sin \delta\left(k h-K_{2}\right)\right\} \exp (-k h)$

for a strike-slip source.

The excitation spectra for dip-slip and strike-slip sources on a vertical fault $\left(\delta=90^{\circ}\right)$ calculated (a) on the basis of modal theory and (b) according to Eqs. (26) and (27) are compared in Fig. 4. The difference between the two approaches is negligible and grows slightly with increasing thickness of the water layer.

Equations (26) and (27) allow us to compare the maximum spectral amplitude of tsunamis generated by different sources and at different strike-receiver angles. The following conclusions may be easily drawn:

- When the azimuth is perpendicular to strike $\left(\phi=0^{\circ}\right)$, the maximum spectral amplitude of tsunamis generated by a dip-slip (or thrust) mechanism does not depend on the dip angle $\delta$ : the excitation function is equal to $2 i F k^{2} h \exp (-k h) \exp (-2 i \delta)$.

- At shallow focal depths, a dip-slip mechanism generates the most intensive tsunami in the direction of strike $\left(\phi=90^{\circ}\right)$. The excitation function at this angle is equal to $F k \sin 2 \delta\left(k h+K_{2}\right) \exp (-k h)$. Thus the greatest tsunamigenic effect is caused by a thrust along a fault dipping at $45^{\circ}$, in the direction perpendicular to the strike of the fault. This conclusion is in agreement with that obtained by Okal (1988) who calculated synthetic mareograms at different azimuths considering the largest peak-to-peak amplitude value.

- At shallow focal depths, if compared to dip-slip along a sloping fault, dip-slip mechanism along a vertical fault $\left(\delta=90^{\circ}\right)$ generates negligible tsunamis: the excitation function is $2 F k^{2} h \exp (-k h) \cos \phi$. This conclusion is also in good agreement with Okal's results.

- Unlike the dip-slip case, the maximum spectral amplitude of tsunami from shallow strike-slip sources, along normal fault $\left(\delta=90^{\circ}\right)$, does not vanish.

\section{Tsunami excitation in a semi-infinite water layer}

Now we apply the representation theorem for studying tsunami generation in a semi- infinite water layer with sloping boundary (Fig. 5). Since there is no exact solution for the normal displacement of the non-planar surface of the elastic half-space, we assume the source depth to be much larger that the water layer, so that the deviation of the surface of the 


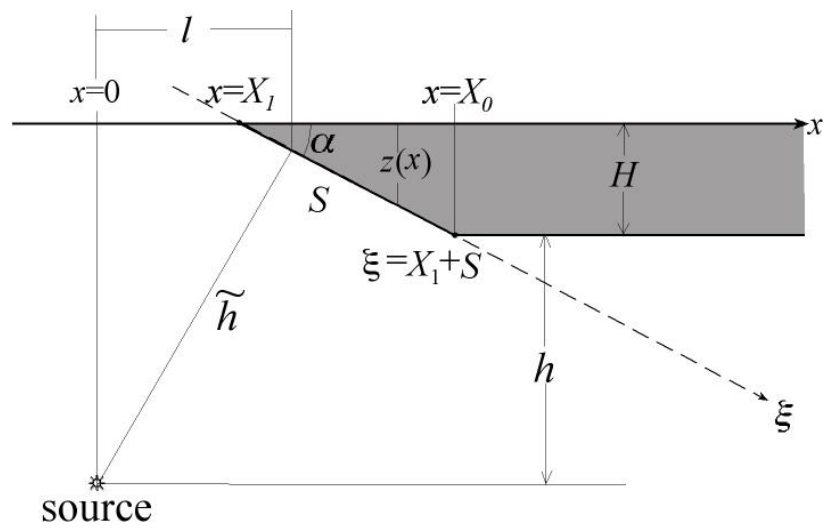

Fig. 5. Scheme of a semi-infinite water layer (with thickness $H$ ) with a sloping $\left(0^{\circ}<\alpha<90^{\circ}\right)$ boundary: the bottom consists of two segments - the horizontal one, from $x=X_{0}$ to $\infty$, and the sloping one, from $\xi=X_{1}$ to $\xi=X_{1}+S$, where $S$ is the length of the oceanic rise.

elastic half-space from a plane is small compared with the source depth. Then the normal displacement of such surface should be approximately the same as that of the plane surface coinciding with the non-planar surface at each segment. Figure 5 illustrates this approximation: the bottom consists of two segments - the horizontal one, from $x=X_{0}$ to $\infty$, and the sloping one, from $\xi=X_{1}$ to $X_{1}+S$, where $S$ is the length of the shelf.

We shall consider tsunami waves only on the $x$-axis, which is the axis of symmetry. Strike-slip mechanisms are not considered in this case since along the $x$-axis the tsunami intensity vanishes.

Integration in the representation theorem should be performed now over the horizontal boundary $z=H, X_{0}<x<$ $\infty, \infty<y<\infty$, and over the sloping part of the boundary (oceanic rise) $X_{1} \leq \xi \leq X_{1}+S, 0 \leq z \leq H$.

If we use the Green function (15) for the infinite layer, the normal component of displacement at the oceanic rise does not vanish, and we must use the representation (7). The normal component of displacement on the sloping boundary should differ from that on the infinite horizontal plane. However, if we assume the normal displacement on the oceanic rise to be the same as that on the infinite plane boundary shown in Fig. 6 by the dashed line, the normal stress, $\tau_{n}$, on this part of the boundary is equal to zero. Using this assumption we can eliminate the term $P u_{n}^{q}$ in the integrand in (7), since, on this part, $\tau_{n}=P=0$.

At first we shall consider the contribution to the tsunami field from the displacement of the horizontal boundary. For brevity we denote the vertical displacement of the boundary, which is given by (A5), as $W(x, y)$. Since the observation point $M$ (see Fig. 2 ) is on the $x$-axis at a distance $r$ from the projection on the surface of the source, the distance from $\mathrm{M}$ to a (current) point in the vicinity of the source (where $W(x, y)$ does not vanish) is approximately $r-x$ (see Eqs. (17) and Fig. 2). Then the contribution to the tsunami field from the horizontal boundary may be written as:

$$
\begin{aligned}
& W_{h o r}(r, 0)=\exp (-\pi i / 4) \exp (-i k r) \\
& \frac{2 k \sinh k H}{H+\frac{g}{\omega^{2}} \sinh ^{2} k H} \sqrt{\frac{1}{8 \pi k r}} \\
& \int_{X_{0}}^{\infty} \exp (i k x) d x \int_{-\infty}^{\infty} W(x, y) d y .
\end{aligned}
$$

The inner integral is calculated in closed form:

$$
\begin{aligned}
& \int_{-\infty}^{\infty} W(x, y) d y=\frac{1}{\pi \rho \beta^{2}} \\
& \frac{2 h^{2} x \cos 2 \delta+h\left(h^{2}-x^{2}\right) \sin 2 \delta}{\left(x^{2}+h^{2}\right)^{2}} \equiv \Phi(x, h, \delta)
\end{aligned}
$$

while the integral

$$
\int_{X_{0}}^{\infty} \Phi(x, h, \delta) \exp (i k x) d x
$$

is calculated numerically.

The contribution from the sloping boundary is estimated in a similar way: due to the non-horizontal alignment of the boundary the integral over $y$ becomes $\Phi(\xi, \tilde{h}, \delta+\alpha)(\tilde{h}$ and $\alpha$ are shown in Fig. 5). In the expression for the Green function (15) the multiplier $\cosh k(H-z(\xi))$ must be taken into account, and $x$ in the exponent must be replaced by $l+\xi \cos \alpha$ ( $l$ and $z(\xi)=\left(\xi-X_{1}\right) \sin \alpha$ are also shown in Fig. 5). In conclusion, the contribution due to the sloping boundary is the following:

$$
\begin{aligned}
& W_{\text {slope }} \cong \exp (-\pi i / 4) \exp (-i k r) \frac{2 k \sinh k H}{H+\frac{g}{\omega^{2}} \sinh ^{2} k H} \sqrt{\frac{1}{8 \pi k r}} \\
& \int_{Q_{1}}^{Q_{1}+S} \Phi(\xi, \tilde{h}, \delta+\alpha) \exp (i k(L+\xi \cos \alpha)) \cosh k(H-z(\xi)) d \xi .(31)
\end{aligned}
$$

Figure 6 shows the results of the numerical calculation of the excitation spectra for different locations of the source with respect to the coastline and for different source depths. For comparison the excitation spectra for an infinite water layer, with thickness $H$, are shown as well.

If the source is located under the water at a distance from the coastline equal (or larger) than the source depth, the excitation function differs negligibly from that for the case of an infinite water layer, i.e. the shore practically does not affect the excitation of tsunami. When the source is located closer to the coastline, the content of low frequencies in the excitation spectrum decreases, while the content of high frequencies dramatically increases. When the source is located under the land at a distance equal to the source depth, the maximum in the excitation spectrum becomes less than $10 \%$ of that radiated if the same source is located in the open ocean. 

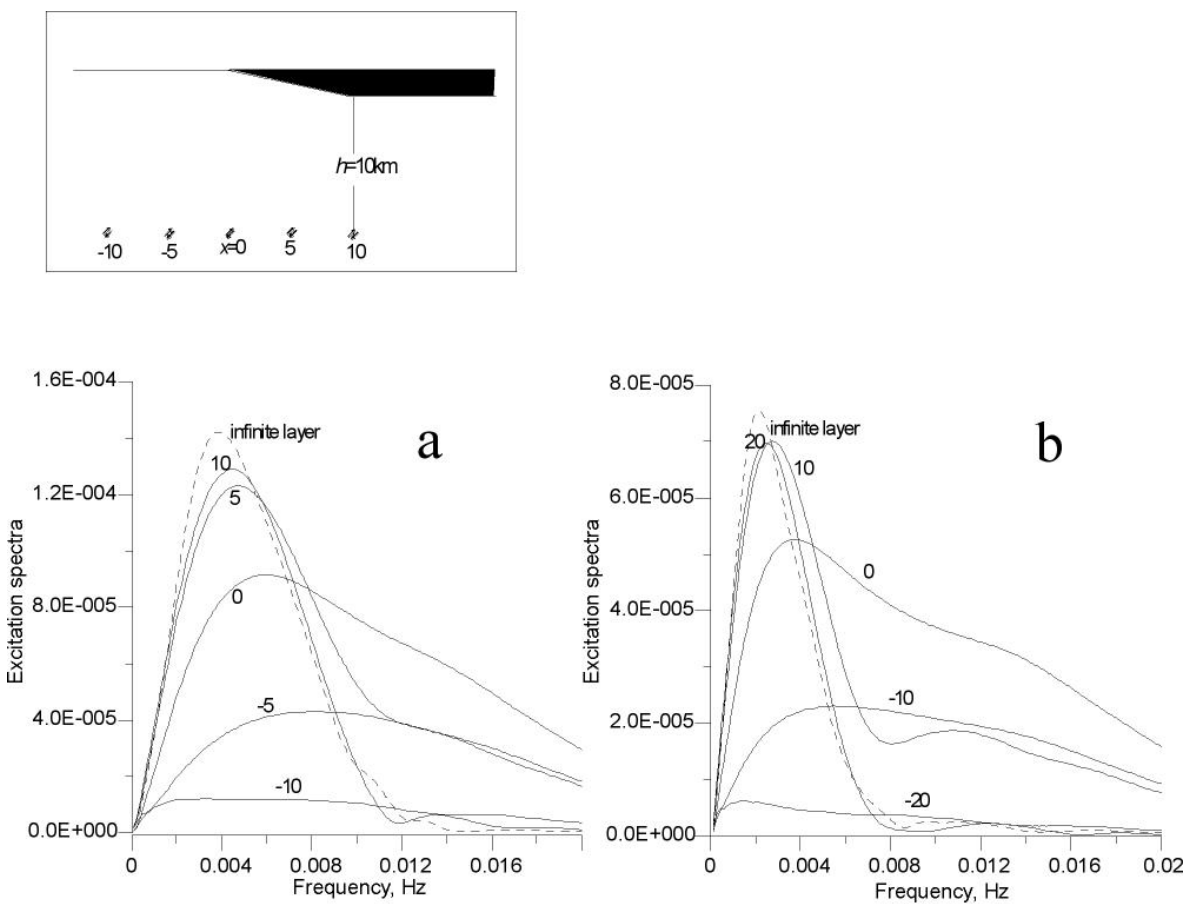

Fig. 6. Excitation spectra from point sources $\left(\delta=45^{\circ}\right.$ - thrust) located at different distances from the coastline indicated by the numbers on the curves (negative values correspond to inland sources): (a) $h=10 \mathrm{~km}$, (b) $h=20 \mathrm{~km}$. The geometry of the model and the location of the sources for $h=10 \mathrm{~km}$ are drawn in the uppermost left panel. The water layer is $2 \mathrm{~km}$ thick.

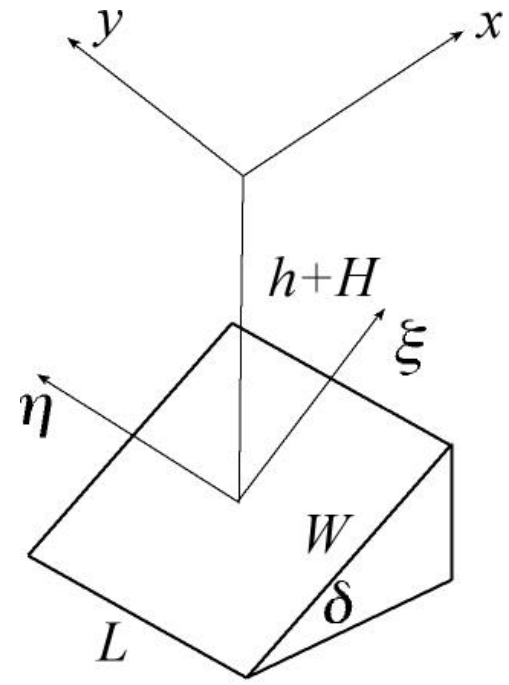

Fig. 7. Scheme of a finite rectangular fault: an elementary source is located at the point $(\xi, \eta)$ in the fault system, which has the coordinates $(x-\xi \cos \delta, y-\eta,(h+H)-\xi \sin \delta)$.

\section{Tsunami excitation from a finite rectangular source}

Observed tsunamis are generated only by large earthquakes of approximately magnitude 6 or greater. For such earthquakes the faults dimensions are large, and the real seismic source cannot be modelled by a point source: it is necessary to take into account the finite size of the fault.
To compare tsunamis generated by point and finite sources it is sufficient to compare the excitation functions. The finite source may be represented as a superposition of point sources distributed over the area of the fault. We shall consider a rectangular fault area with length $L$ and width $W$, assuming slip either perpendicular to strike (dip-slip) or in the direction of strike (strike-slip). The geometry of the fault and the coordinates $(\xi, \eta)$ related to the fault plane are shown in Fig. 7. At first we consider a model with an infinite water layer.

An elementary source located at the point $(\xi, \eta)$ in the fault system has the coordinates $(x-\xi \cos \delta, y-\eta, h-\xi \sin \delta)$. Now it is sufficient to replace in the part of the expression for the displacement $\mathbf{U}$ that depends on $r$ and $h$ (i.e. in $\chi \exp (-i k r))(x, y, h)$ by $(x-\xi \cos \delta, y-\eta, h-\xi \sin \delta)$ and to integrate over $\xi$ from $-L / 2$ to $L / 2$, and over $\eta$ from $-W / 2$ to $W / 2$. If we want to compare the results with a point source with the same seismic moment, we must divide the result of integration by the area $L W$.

Assuming that the distance $r$ is much larger than the source size, we may replace $r$ in the exponent by $r-\xi \cos \delta \cos \phi-$ $\eta \sin \phi$. Then, after integration, we obtain the expression for the excitation function in one and the same form for both dip-slip and strike-slip:

$$
\begin{aligned}
& \frac{2 \sin (k W \sin \phi / 2)}{k W \sin \phi} \\
& \left\{\frac{A \gamma-B}{\gamma^{2}} \frac{2 \sinh (\gamma L / 2)}{L}+\frac{B}{\gamma} \cosh (\gamma L / 2)\right\},
\end{aligned}
$$




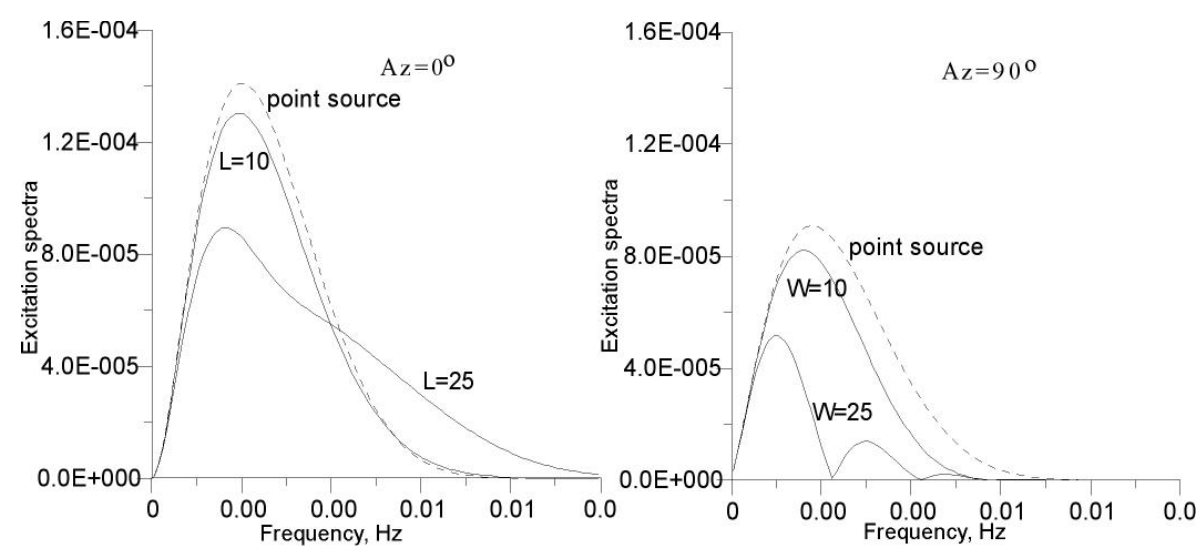

Fig. 8. Excitation spectra for point (dashed lines) and finite sources (solid lines) for thrust $\left(\delta=45^{\circ}\right)$ at two azimuths ( $A z$ ). Depth of source is $10 \mathrm{~km}$. Excitation spectra at $A z=0^{\circ}$ do not depend on the width, $W$, of the fault, whereas at $A z=90^{\circ}$ the dependence on length, $L$, of the fault is negligible.

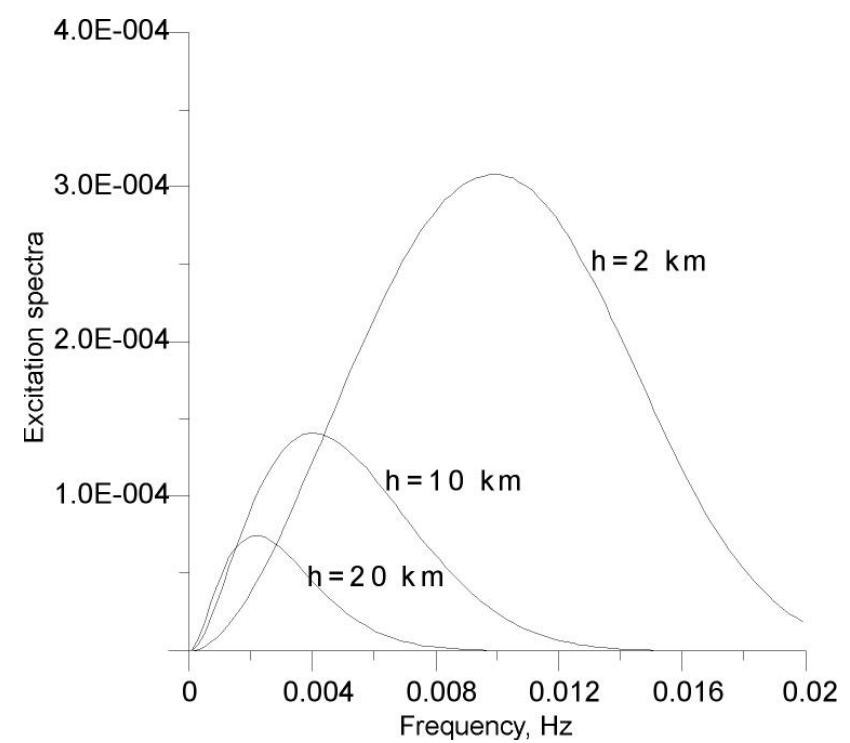

Fig. 9. Excitation spectra at $A z=0^{\circ}$ for point sources $\left(\delta=45^{\circ}\right.$ thrust) at different depths.

where

$\gamma=k \sin \delta+i k \cos \delta \cos \phi$.

The coefficients $A$ and $B$ are different for dip-slip:

$A=F k\left[2 i k h \cos 2 \delta \cos \phi+2 k h \sin 2 \delta+\sin ^{2} \phi \sin 2 \delta\left(K_{2}-k h\right)\right] \exp (-k h)$
$B=F k\left[-2 i k \sin \delta \cos 2 \delta \cos \phi-2 k \sin \delta \sin 2 \delta+k \sin ^{2} \phi \sin \delta\right] \exp (-k h)$

and for strike-slip:

$A=F k\left[-2 i k h \cos \delta \sin \phi+\sin \delta \sin 2 \phi\left(k h-K_{2}\right)\right] \exp (-k h)$

$B=F k^{2} \sin \delta\left[i \sin \phi \sin 2 \delta-\sin ^{2} \delta \sin 2 \phi\right] \exp (-k h)$

Figure 8 shows the excitation spectra for point and finite thrust sources. The main peculiarity of the excitation spectra for extended sources as compared with that for point sources is that, in general, for a given scalar moment, the spectral level, as well as the maximum spectral amplitude, decreases with increasing fault size, in agreement with the results found by Ward (1982), who adopted the modal approach and considered a line source. At the same time, the high-frequency content increases with fault length increasing in the direction perpendicular to the strike of the fault plane $\left(A z=0^{\circ}\right)$. This phenomenon follows from the dependence of the excitation spectra on the source depth (Fig. 9): shallow point sources generate intense high-frequency tsunami; with increasing source depth the spectral maximum decreases and is shifted to lower frequencies. Hence, if the upper edge of the fault approaches the bottom of the water layer, this part of the fault generates intense high-frequency wave (e.g. greater than $0.01 \mathrm{~Hz}$ ), whereas the lower edge radiates in the lowfrequency range and excites much less intense wave.

In the case of a semi-infinite water layer, the integration over the fault area cannot be performed in a closed form. As in the case of the point source, we shall treat the tsunami field generated by a thrust in a point on the $x$-axis. In analogy with the case of the infinite water layer, the effect of the fault size derives from the length of the fault rather than from its width. Therefore we may limit the analysis to a linear source in the $x z$-plane. As before, we assume the direction of slip to coincide with the orientation of the fault. The integration along the fault is performed numerically.

Figure 10 shows the excitation functions for the same model and source locations as in Fig. 6, but for a fault length $L=25 \mathrm{~km}$. As in the case of the infinite water layer, a significant difference between the point and the finite source is observed for the shallow earthquake $(h=10 \mathrm{~km})$, for which the upper edge of the fault is close to the bottom of the water layer. The maximum of the spectrum becomes lower for offshore and higher for inland sources. For the intermediate depth crustal earthquake $(h=20 \mathrm{~km})$, the influence of the source length on the excitation spectrum is much weaker than when $h=10 \mathrm{~km}$. 


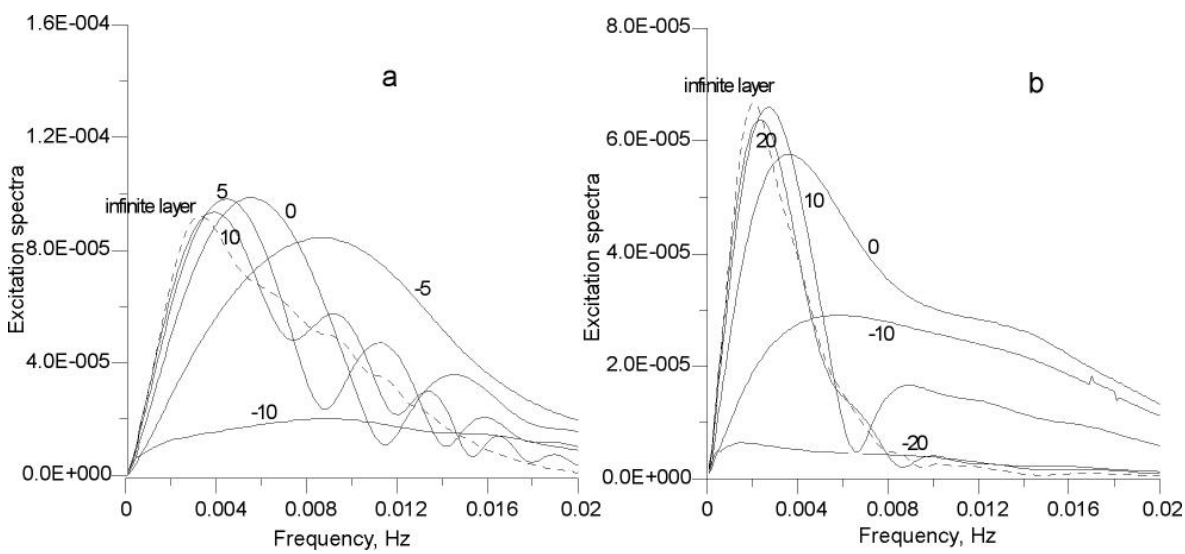

Fig. 10. Excitation spectra for the same model as in Fig. 6, but for a finite source with $L=25 \mathrm{~km}$. (a) $h=10 \mathrm{~km}$; (b) $h=20 \mathrm{~km}$.

Inasmuch as the strongest effect of the source size arises when a part of the fault approaches to the bottom of the liquid, an influence of the fault dip on the excitation spectrum should be expected. Figure 11 shows the excitation spectra for three dips of the fault situated at the same depth ( $h=15 \mathrm{~km}$ ), where $h$ is defined in Figs. 5 and 7 (i.e. is the distance from the bottom of the liquid layer to the middle point of the fault) and with the same length $(L=25 \mathrm{~km})$. An increase of the dip leads to an increase of the tsunami maximum spectral amplitude from the offshore earthquakes and a decrease of tsunami intensity from inland earthquakes. Thus, inland earthquakes with a shallow dipping thrust mechanism can generate tsunamis.

\section{Synthetic mareograms}

It is difficult to compare tsunamigenic effects from earthquakes with different geometric parameters on the basis of the excitation spectra: more grounded conclusions can be drawn from the comparison of synthetic mareograms. The Green function method does not allow the mareograms to be calculated in the zone for which $r<h$ and $k r<5$, but tsunami waveforms can be compared for different earthquake models and source locations using the mareograms calculated at the same distance from the source. This is of practical interest to determine tsunami waves generated by inland/coastal earthquake and propagating to distant coasts (e.g. an island or a transoceanic coastline). The mareograms calculated for the same model as in Fig. 11 are shown in Fig. 12 (the high-frequency oscillations are computational noise due to the numerical integration, and they should not be taken into consideration). Consistent with Fig. 11, slip along a steep fault generates a more intensive tsunami. A source located under the coast (position 3 ) in the case of slightly sloping fault generates tsunami of the same intensity as an offshore earthquake of the same mechanism and depth. In position $1(10 \mathrm{~km}$ inland) the intensity of tsunami is about $25 \%$ of that from off-shore earthquake.
Thus, the decay of the tsunami intensity when moving the source toward shore depends on dip, length and depth of the fault. It is reasonable to estimate the tsunami intensity as the maximum peak-to-peak amplitude, calculated from the synthetic mareograms. Figures 13a, b show the normalized maximum tsunami amplitude versus distance $d$ from the coast (negative values correspond to inland sources). On the average the rate of the amplitude decay is slower with increasing fault length (see Fig. 13b) and with increasing source depth (compare 13a and 13b). The data for two source depths (10 and $30 \mathrm{~km}$ ) are drawn together as a function of $d / h$, and the average curve is calculated on the basis of all computed tsunamis. On the basis of this figure we may conclude that, on the average, the tsunami amplitude from inland source at a distance from the coast equal to the source depth becomes 4-5 times less than that from a source in the open ocean. If the source is located under the coastline, the intensity of tsunami is approximately the same as from oceanic sources (e.g. 1964 Alaska earthquake).

\section{Discussion and conclusions}

We may draw an analogy between the generation of tsunami by inland/coastal seismic sources and the diffraction of seismic waves in the vicinity of a shadow zone. If we consider the ray theory, the boundary of a shadow zone should be sharp, and no waves should be observed as soon as this boundary is crossed. However, it is well known that in the vicinity of the boundary between the shadow and illuminated zones we observe diffracted waves, which attenuate gradually toward the shadow. The generation of diffracted waves is explained by the Huygens principle: each point reached by the wave becomes a source of secondary waves radiating in all directions. Mathematically, the Huygens principle is formalized in the framework of the Green function method. A similar situation arises with the generation of tsunami waves. According to the modal theory, tsunamis are not generated as soon as the source is moved inland, because the tsunami mode does not exist without a gravitating water layer. So 

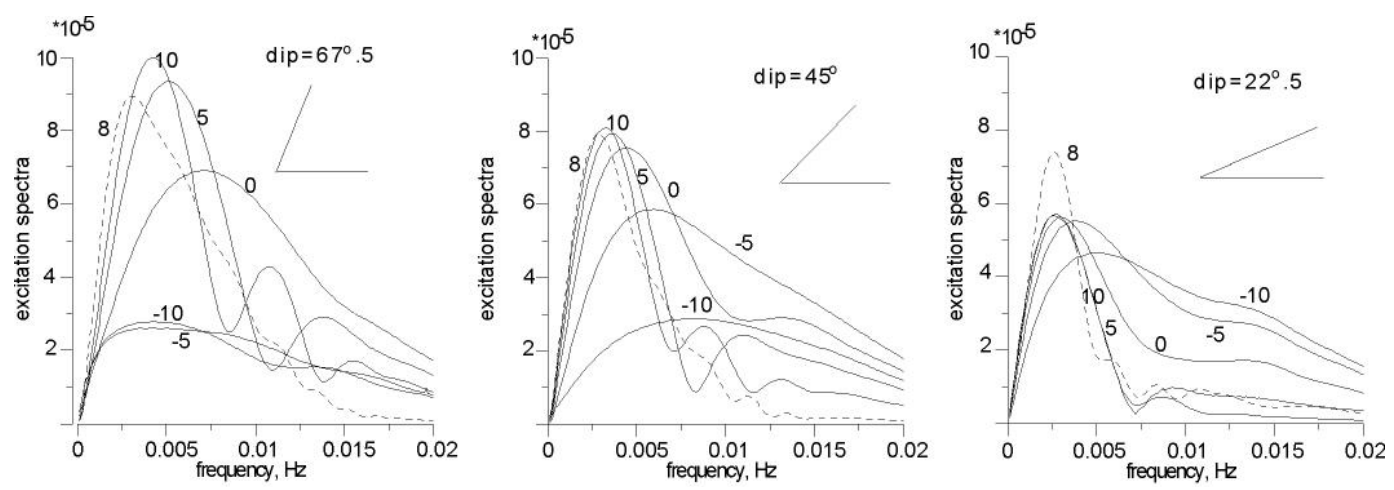

Fig. 11. Excitation spectra for different fault dips. Source depth is $h=15 \mathrm{~km}$, and fault length $L=25 \mathrm{~km}$. Dip angles are indicated in the corresponding graphs. Dashed lines correspond to the case of an infinite water layer. The thickness of the water layer is $2 \mathrm{~km}$.

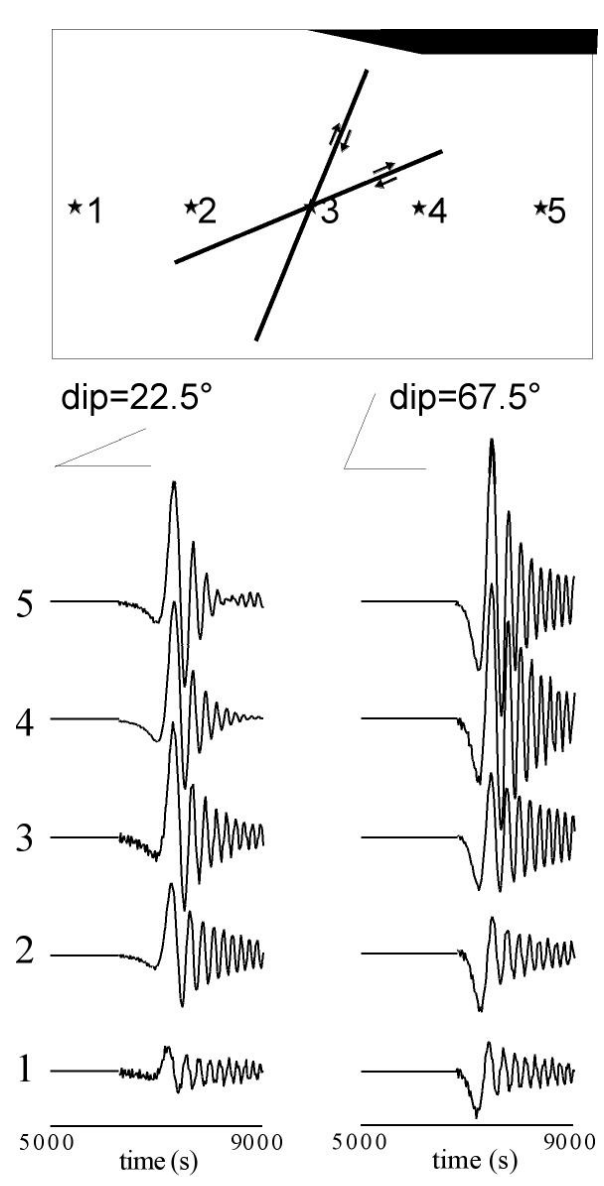

Fig. 12. Synthetic mareograms calculated at a distance $\Delta=$ $1000 \mathrm{~km}$ from the coast excited by the sources whose location is shown in the panel above.

if we consider the modal theory, a coastline should play the same role as the boundary of a shadow zone: as long as the source is under the water layer the tsunamis are generated, but when the source is moved under the land the tsunami disappears. Obviously it is not so. Generation of tsunami should decay gradually when the inland source distance increases.
But the question is: how fast? To answer this question it was natural to apply the same approach applied in the analysis of diffracted waves, i.e. the Green function technique. The application of this approach allowed us to get some rough estimates of the decay of tsunami amplitude as the earthquake source moves inland from the coastline.

We did not estimate tsunami amplitude or run-up at the local coast: our estimates are valid for tsunami waves sufficiently far away from the source $(r>>h$ and $k r=5)$. Also, the waves generated by inland sources should propagate from the coast rather than toward the coast. For estimating the effects near the coast the linear theory fails; in this case we must solve the non-linear equations for the near field, and this can be done only by the use of numerical methods. Our goal is to show that not only offshore, but also inland earthquakes in the vicinity of the coastline may generate tsunamis. According to our estimates, the tsunami may be sufficiently intensive if the inland epicentral distance from the coast is of the same order as the focal depth.

The main conclusions that can be drawn from our modelling are as follows:

- the dependence of the tsunami intensity on the fault size is different for offshore and coastal earthquakes: for offshore sources, tsunami amplitude increases with decreasing fault size (i.e. greater stress drop); for inland/coastal sources the effect is opposite;

- the relative content of high frequencies in the tsunami signal for an offshore source increases with decreasing distance of the source from the shore;

- as the source moves inland from the coastline, the rate of the tsunami amplitude decay depends on the source depth and on the dip of the fault. On the average, when the source is located inland at a distance comparable with the source depth, the tsunami amplitude is about $20-25 \%$ of that generated by a offshore earthquake at a distance larger than the source depth. conclusions for a quick and efficient tsunami hazard assessment. 


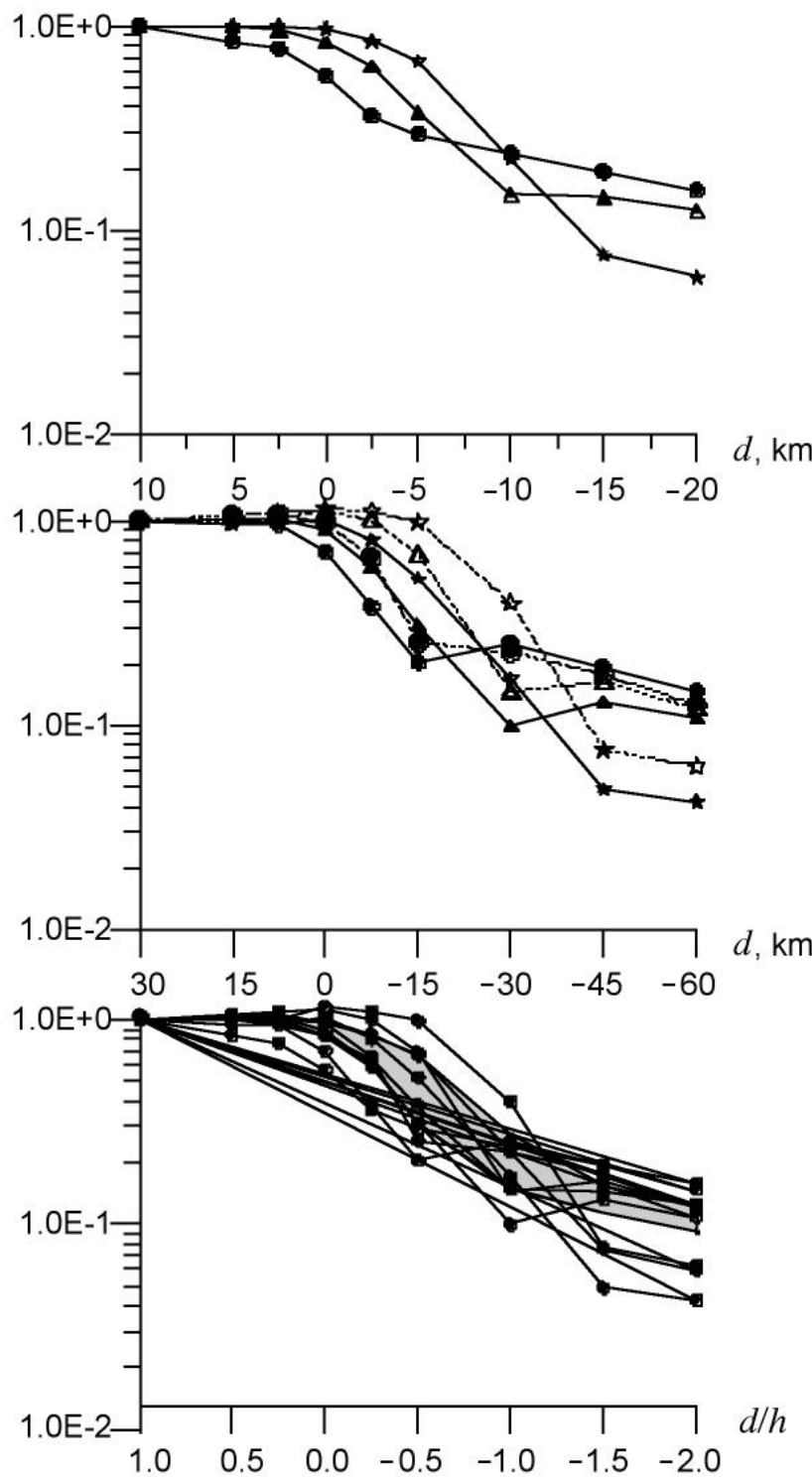

Fig. 13. Normalized maximum tsunami amplitude: decay versus the distance $d$ from the coastline. (a) $h=10 \mathrm{~km}, L=20 \mathrm{~km}$. Circles correspond to $\delta=60^{\circ}$, triangles to $\delta=45^{\circ}$, stars to $\delta=$ $30^{\circ}$. (b) $h=30 \mathrm{~km}, L=20 \mathrm{~km}$ (bold lines), $L=60 \mathrm{~km}$ (dashed lines). (c) The data from (a) and (b) versus $d / h$. The Boundaries of the shaded area correspond to the mean value $\pm r m s$ error.

The analysis of how earthquake source parameters affect the excitation of the tsunami wavefield has a great importance in the study of both local and distant tsunamis that may generate great amplitude run-up heights. Far-field estimates allows us to draw useful conclusions for a quick and efficient tsunami hazard assessment.

\section{Appendix A}

Let be $K_{1}=\frac{\alpha^{2}}{\alpha^{2}-\beta^{2}}, K_{2}=\frac{\beta^{2}}{\alpha^{2}-\beta^{2}}$, where $\alpha$ and $\beta$ are Pand S-wave velocities, respectively. The coordinates of the source and of the observation point are $(0,0, h)$ and $(x, y, 0)$, respectively, $r^{2}=x^{2}+y^{2}, R^{2}=x^{2}+y^{2}+h^{2}, \cos \varphi=\frac{x}{r}$.

The vertical $(W)$ and horizontal $(U)$ displacements are:

- for a vertical force:

$$
\begin{aligned}
& W=\frac{1}{4 \pi \rho \beta^{2} R}\left(K_{1}+\frac{h^{2}}{R^{2}}\right) \\
& U_{r}=\frac{1}{4 \pi \rho \beta^{2} R}\left(\frac{r h}{R^{2}}+K_{2} \frac{R-h}{R}\right)
\end{aligned}
$$

- for a horizontal force acting along the $\mathrm{x}$-axis:

$$
\begin{aligned}
& W=\frac{\cos \phi}{4 \pi \rho \beta^{2} R}\left(\frac{r h}{R^{2}}-K_{2} \frac{R-h}{r}\right) \\
& U_{x}=\frac{1}{4 \pi \rho \beta^{2} R}\left(\frac{R^{2}+x^{2}}{R^{2}}+K_{2} \frac{\left(y^{2}+h^{2}+R h\right)(R-h)^{2}}{r^{4}}\right) \\
& U_{y}=\frac{x y}{4 \pi \rho \beta^{2} R}\left(\frac{1}{R^{2}}-K_{2} \frac{(R-h)^{2}}{r^{4}}\right)
\end{aligned}
$$

- for a dip-slip mechanism on a fault with strike along the y-axis and $\operatorname{dip} \delta$ :

$$
\begin{aligned}
& W=\frac{1}{4 \pi \rho \beta^{2} R}\left\{\frac{6 h^{2} x}{R^{4}} \cos 2 \delta+\left(\frac{3 h\left(h^{2}-x^{2}\right)}{R^{4}}+\right.\right. \\
& \left.\left.K_{2}\left(\frac{y^{2} h}{r^{2} R^{2}}-\frac{(R-h)\left(y^{2}-x^{2}\right)}{r^{4}}\right)\right) \sin 2 \delta\right\} \\
& U_{x}=\frac{1}{4 \pi \rho \beta^{2} R}\left\{\frac{6 h^{2} x}{R^{4}} \cos 2 \delta+\frac{x}{R^{2}}\left(\frac{3\left(h^{2}-x^{2}\right)}{R^{2}}-\right.\right. \\
& \left.\left.K_{2}\left(\frac{y^{2}(3 R+h)-R^{2}(R+h)}{(R+h)^{3}}\right)\right) \sin 2 \delta\right\}
\end{aligned}
$$

\section{Appendix B}

In the incompressible liquid layer one has:

$$
P(k, z)=A \exp (k z)+B \exp (-k z)
$$

$$
\begin{aligned}
& W(k, z)=-\frac{1}{\rho_{f} \omega^{2}} \frac{d P}{d z}=\frac{k}{\rho_{f} \omega^{2}}(-A \exp (k z)+B \exp (-k z)) \\
& U(k, z)=\frac{k}{\rho_{f} \omega^{2}} P=\frac{k}{\rho_{f} \omega^{2}}(A \exp (k z)+B \exp (-k z))
\end{aligned}
$$

and using the boundary condition at the water surface, the coefficients $A$ and $B$ may be replaced by one unknown coefficient $Q$ :

$$
A=-Q\left(1-\frac{g k}{\omega^{2}}\right) \quad B=Q\left(1+\frac{g k}{\omega^{2}}\right)
$$


In the solid half-space one has:

$U=\frac{i \alpha^{2}}{\omega c} C \exp \left(-\frac{\omega r_{\alpha}(z-H)}{\alpha}\right)-\frac{i r_{\beta}}{\omega} E \exp \left(-\frac{\omega r_{\beta}(z-H)}{\beta}\right)$

$W=\frac{\alpha r_{\alpha}}{\omega} C \exp \left(-\frac{\omega r_{\alpha}(z-H)}{\alpha}\right)-\frac{\beta^{2}}{\omega c} E \exp \left(-\frac{\omega r_{\beta}(z-H)}{\beta}\right)$

where $r_{\alpha}=\sqrt{\frac{\alpha^{2}}{c^{2}}-1} \quad r_{\beta}=\sqrt{\frac{\beta^{2}}{c^{2}}-1}, \alpha$ and $\beta$ are the Pand $\mathrm{S}$-wave velocities in the half-space, $c=\frac{\omega}{k}$ is the phase velocity of tsunami wave (Panza et al., 2000). From $\tau_{x z}=0$ at $z=H$ it follows:

$\frac{2 \alpha r_{\alpha}}{c} C=\left(2 \frac{\beta^{2}}{c^{2}}-1\right) E$.

From the continuity of the normal displacement at $z=H$ we can express the coefficient $E$ in terms of $Q$ :

$E=-\frac{4 Q}{\rho_{f} c^{2}}\left(\cosh k H-\frac{g k}{\omega^{2}} \sinh k H\right)$

and from the continuity of the normal stress, taking into account that $c / \beta \ll 1, c / \alpha \ll 1$, one obtains:

$E=\frac{2 K_{1} Q}{\rho \beta^{2}}\left(-\sinh k H+\frac{g k}{\omega^{2}} \cosh k H\right)$,

where $\rho$ is the density of the elastic half-space.

From (B5) and (B6) we obtain the dispersion equation for the system formed by an incompressible water layer over an elastic half-space:

$\cosh k H-\frac{g k}{\omega^{2}} \sinh k H=\frac{K_{1} \rho_{f} c^{2}}{2 \rho \beta^{2}}\left(\sinh k H-\frac{g k}{\omega^{2}} \cosh k H\right)$.

From (B1), the vertical displacement of the water surface is:

$W(k, 0)=\frac{2 Q k}{\rho_{f} \omega^{2}}$.

Using the expressions for the vertical and horizontal displacements in the water layer (B1) and in the solid half-space (B3), and taking into account the relationships between the coefficients (B2), (B4) and (B5), we calculate the energy integral as a sum of integrals over the liquid layer and the solid half-space:

$I_{0}^{l i q}=\rho_{f} \int_{0}^{H}\left(U^{2}+W^{2}\right) d z \cong \frac{4 k Q^{2}}{\rho_{f} \omega^{4} \sinh k H}$

$I_{0}^{s o l} \cong \frac{Q^{2} K_{1}^{2}}{\rho \omega^{4} k H} \frac{c^{2} g}{\beta^{4}}$.

Remembering that $c / \beta \ll 1$ we may write an approximate expression for the total energy integral as follows:

$I_{0}=\frac{Q^{2} k}{\omega^{4} \sinh ^{2} k H}\left(\frac{c^{4}}{\rho \beta^{4}}+\frac{4 \sinh k H}{\rho_{f}}\right) \cong \frac{4 k Q^{2}}{\rho_{f} \omega^{4} \sinh k H}$

thus obtaining:

$\frac{W(k, 0)}{\sqrt{c u I_{0}}}=\frac{\sqrt{k \sinh k H}}{\sqrt{\rho_{f} c u}}$.
Acknowledgements. We acknowledge the useful comments and the critical review of an anonymous reviewer and of E. L. Geist. We acknowledge financial support from the 2000 Italian MIUR-Cofin Project.

\section{References}

Ben-Menahem, A. and Rosenman, M.: Amplitude patterns of tsunami waves from submarine earthquake, J. Geophys. Res., 77, 3097-3128, 1972.

Comer, R. P.: The tsunami mode of a flat Earth and its excitation by earthquake sources, Geophys. J. Astr. Soc., 77, 1-28, 1984a.

Comer, R. P.: Tsunami generation: a comparison of traditional and normal mode approach, Geophys. J. Astr. Soc., 77, 29-41, 1984b.

Geist, E. L.: Local tsunamis and earthquake source parameters, Advances in Geophysics, 39, 117-209, 1999.

Hammack, J. L.: A note on tsunamis: their generation and propagation in an ocean of uniform depth, J. Fluid Mechanics, 60, 769-799, 1973.

Johnson, J. M., Satake, K., and Holdahl, S. R.: The 1964 Prince William Sound earthquake: Joint inversion of tsunami and geodetic data, J. Geophys. Res., 101, 523-532, 1996.

Kajiura, K.: The leading wave of tsunami, Bull. Earthq. Res. Inst., 41, 535-571, 1963.

Kajiura, K.: Tsunami energy in relation to parameters of earthquake fault model, Bull. Earthq. Res. Inst. Univ. Tokyo, 56, 415-440, 1981.

Lee, J. J. and Chang, J. J.: Water waves generated by an impulsive bed upthrust of a rectangular block, Appl. Ocean Res., 2, 165170,1980

Levshin, A. L., Yanovskaya, T. B., Lander, A. V., et al.: Seismic surface waves in laterally inhomogeneous Earth, Keilis-Borok editor, Kluwer, Dordrecht, 1989.

Maruyama, T.: Static elastic dislocation in an infinite and semiinfinite medium, Bull. Earthq. Res. Inst., Tokyo Univ., 42, 289368, 1964.

Okada, Y.: Surface deformation due to shear and tensile faults in a half-space, Bull. Seism. Soc. Am., 75, No. 4, 1135-1154, 1985.

Okal, E. A.: Mode-wave equivalence and other asymptotic problems in tsunami theory, Phys. Earth and Planet. Inter., 30, 1-11, 1982.

Okal, E. A.: Seismic parameters controlling far-field tsunami amplitudes: a review, Natural Hazards, 1, 67-96, 1988.

Ortiz, M., Singh, S. K., Pacheco, J., and Kostoglodov, V.: Rupture length of the October 9, 1995 Colima-Jalisco earthquake (Mw 8) estimated from tsunami data, Geophys. Res. Lett., 25, $2857-$ 2860, 1998.

Panza, G. F., Schwab F. A., and Knopoff, L.: Multimode surface waves for selected focal mechanisms. I. Dip-slip sources on a vertical fault plane, Geophys. J. R. Astr. Soc., 34, 265-278, 1973.

Panza, G. F., Romanelli, F., and Yanovskaya, T. B.: Synthetic tsunami mareograms for realistic oceanic models, Geophys. J. Int., 141, 498-508, 2000.

Pod'yapollsky, G. S.: Generation of longperiodic gravitational wave in the ocean by seismic source in the crust, Izvestia AN SSSR, Fizika Zemli, 1, 7-24, 1968.

Titov, V. V. and Synolakis, C. E.: Extreme inundation flow during the Hokkaido-Nansei-Oki tsunami, Geophys. Res. Lett., 24, 11, 1315-1318, 1997. 
Ward, S. N.: Relationship of tsunami generation and an earthquake source, J. Phys. Earth, 28, 441-474, 1980.

Ward, S. N.:On tsunami nucleation II. An instantaneous modulated line source, Phys. Earth Planet. Int., 27, 273-285, 1982.
Yamashita, T. and Sato, R.: Generation of tsunami by a fault model, J. Phys. Earth, 22, 415-440, 1974.

Yamazaki, K.: Theory of crustal deformation due to dilatancy and quantitative evaluation of earthquake precursors, Sci. Rep. Tohoku Univ., Ser. 5 Geophys., 25, 115-167, 1978. 